\title{
Correlation of PD-L1 Expression with Histological Patterns and Treatment Response in Non-Small Cell Lung Cancer
}

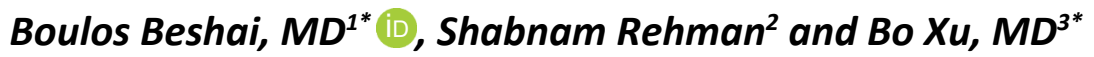 \\ ${ }^{1}$ Department of Pathology, Roswell Park Comprehensive Cancer Center, USA \\ ${ }^{2}$ Department of Internal Medicine/Hematology Oncology, Texas Tech University Health Sciences Center, USA \\ ${ }^{3}$ Department of Pathology, Roswell Park Comprehensive Cancer Center, USA
}

*Corresponding author: Boulos Beshai, MD, Department of Pathology, Roswell Park Comprehensive Cancer Center, Buffalo, NY, USA; Bo Xu, MD, Department of Pathology, Roswell Park Comprehensive Cancer Center, USA

\begin{abstract}
Programmed Death Ligand-1 (PD-L1) inhibitors are currently approved for treatment of advanced stage NonSmall Cell Lung Cancer (NSCLC) patients. We investigated the correlation between PD-L1 expression level in tumor cells by ImmunoHistochemical Method (IHC) to tumor histological growth patterns. Slides or scanned digital images of NSCLC cases tested for PD-L1 between December, 2016 to April, 2018 were blindly re-reviewed to confirm the PD-L1 expression and growth patterns. PD-L1 expression levels were graded as follows: $<1 \%$, negative, $1-50 \%$, low expression, $>50 \%$, high expression. Total 102 cases were identified including 81 cases of adenocarcinoma and 21 cases of Squamous Cell Carcinoma (SCC). Variable expression was seen in SCC; $8 / 21$ cases $(38 \%)$ showed negative expression of PD-L1, 7/21 cases (33\%) showed low expression, and $6 / 21$ cases (29\%) showed high expression. 4/6 total "high-expression" SCC cases (67\%) demonstrated poorly differentiated/non-keratinizing pattern, while $2 / 6(33 \%)$ were well- to Moderately-Differentiated/ keratinizing SCC. In well- to Moderately-Differentiated (WD to MD) adenocarcinoma, $15 / 30$ cases (50\%) showed negative expression of PD-L1, $13 / 30$ cases $(43 \%)$ showed low expression, whereas only $2 / 30$ cases $(7 \%)$ had high expression. On the other hand, moderate- to poorlydifferentiated (MD to PD) adenocarcinomas, 13/51 cases $(26 \%)$ showed negative expression of PD-L1, 15/51 cases $(29 \%)$ showed low expression and $23 / 51$ cases $(45 \%)$ had high expression. $21 / 25$ total "high-expression" cases (84\%) demonstrated poorly-differentiated growth patterns, out of which, 7 cases $(33 \%)$ had a pure solid growth pattern. A 1 -year follow up showed 3 patients with partial or complete response, whose tumors had "high-expression" PD-L1.
\end{abstract}

\section{Keywords}

PD-L1 (Programmed Death Ligand-1), Expression level, NSCLC (Non-Small Cell Lung Carcinoma)

\section{Introduction}

Programmed Death Ligand-1 (PD-L1) proteins are expressed on the cell surface of certain tumor cells, commonly described in Non-Small Cell Lung Carcinoma (NSCLC), melanoma, and gastroesophageal adenocarcinomas. Interaction of PD-L1 on tumor cells with PD-1 receptors on the surface of T-lymphocytes blocks the immune check point, and as a consequence tumor cells escape from being attacked by host immune cells. PD-L1 inhibitors are currently approved by FDA for treatment of advanced stage non-small cell lung cancer patients as either first or second line therapy, based on the degree of protein expression. It is also recommended as either a monotherapy or as part of combined regimen. The PD-L1 expression test was initially performed on advanced stage and metastatic NSCLC. Recently, PD-L1 has been reflex tested on all primary lung cancers, including small cell carcinoma in some practices. Lung adenocarcinoma subtypes and growth patterns have been defined by the 2011 International Association for the Study of Lung Cancer (IASLC) classification, and these criteria were adopted in the 2015 WHO classification [1]. In this study, we investigated the correlation between PD-L1 protein expression level in NSCLC tumor cells detected by Immunohistochemical Method (IHC) to tumor histological growth patterns.

\section{Material and Methods}

Hematoxylin and Eosin (HE) stained slides or

Citation: Beshai B, Rehman S, Xu B (2021) Correlation of PD-L1 Expression with Histological Patterns and Treatment Response in Non-Small Cell Lung Cancer. Int J Cancer Clin Res 8:154. doi. org/10.23937/2378-3419/1410154

Accepted: July 19, 2021: Published: July 21, 2021

Copyright: (c) 2021 Beshai B, et al. This is an open-access article distributed under the terms of the Creative Commons Attribution License, which permits unrestricted use, distribution, and reproduction in any medium, provided the original author and source are credited. 
Table 1: Grading of PD-L1 expression in tumor cells in our institution.

G1 $<1 \%$ positive staining viable tumor cells with both complete and incomplete membranous staining at any intensity level

G2 > or $=1-25 \%$ positive staining viable tumor cells with both complete and incomplete membranous staining at any intensity level

G3 $>$ or $=25-50 \%$ positive staining viable tumor cells with both complete and incomplete membranous staining at any intensity level

G4 $>$ or $=50-75 \%$ positive staining viable tumor cells with both complete and incomplete membranous staining at any intensity level

G5 $>75 \%$ positive staining viable tumor cells with both complete and incomplete membranous staining at any intensity level

scanned digital images of NSCLC cases tested for PD-L1 between December, 2016 to April, 2018 were blindly rereviewed to confirm the level of PD-L1 expression and classification of tumor growth patterns. The antibody used for this study is the FDA approved clone 22C3 from Dako. IHC was performed on an FDA approved "Dako" 48 autostainer. Based on known clinical guidelines of applying the immunotherapy in patient's management options, PD-L1 expression levels were graded as follows: < 1\%, negative (G1), between 1 to $50 \%$, low expression (G2-3), > 50\%, high expression (G4-G5). Further classification is designed in our lab to grade the PD-L1 expression as G1-G5 based on the percentage of tumor cells staining. A positive expression is defined as positive staining viable tumor cells with both complete and incomplete membranous staining at any intensity level (see Table 1). Metastatic cases with unconfirmed lung primary and specimens with insufficient tumor cells were excluded. Cases diagnosed with small cell carcinoma; primary or metastatic, in addition to neuroendocrine neoplasms were excluded. Cytology specimens that had concurrent biopsy or resection with only one single dominant growth pattern, were included. Cytology specimens which had no concurrent biopsy or resection, or the concurrent case had mixed growth patterns, were excluded. In adenocarcinomas, tumors containing lepidic, acinar and papillary patterns are classified as Well-to-Moderately Differentiated (WD to MD). Micropapillary, single cells, signet ring cells and solid patterns are classified As Moderate-to-Poorly Differentiated (MD to PD). Tumors which are confirmed primary lung adenocarcinoma by immunophenotype but lacking any specific growth pattern are classified as poorly-differentiated, NOS (not otherwise specified).

\section{Results}

Total 102 cases were identified, 40 cases were inhouse patients and 62 cases were consults of patients coming to our institution for second opinion or pursuing treatment. Among these, 81 cases of adenocarcinoma and 21 cases of squamous cell carcinoma. The patients' demographics included 61 females and 41 males, with a range of age between 46-89 years-old. Specimens included 13 cytologic cases as fine needle aspiration or endoscopic bronchial ultrasound (4 cases of primary lung cancer, and 9 cases of metastatic tumor), 89 surgical cases; divided into 4 lung wedges, 7 lobectomies, and
78 biopsies ( 53 primary lungcancer, and 25 metastatic tumors). Metastatic locations varied between regional mediastinal lymph nodes, pleura, bone, brain, adrenal gland, liver, and soft tissue. In squamous cell carcinoma, 8 of 21 cases $(38 \%)$ showed negative or $<1 \%$ tumor cells expression of PD-L1, 7 of 21 cases (33\%) showed low expression (1-50\% tumor cells expression), and 6 of 21 cases (29\%) showed high expression (> 50\% tumor cells expression). 4 of 6 total "high-expression" SCC cases (67\%) demonstrated poorly differentiated/ non-keratinizing pattern, while 2 of $6(33 \%)$ were wellto moderately-differentiated/keratinizing SCC. In WD to MD adenocarcinoma, 15 of 30 cases (50\%) showed negative or $<1 \%$ tumor cells expression of PD-L1, 13 of 30 cases $(43 \%)$ showed low expression, whereas only 2 of 30 cases $(7 \%)$ had high expression. On the other hand, MD to PD adenocarcinomas, 13 of 51 cases (26\%) showed negative or $<1 \%$ tumor cells expression of PDL1, 15 of 51 cases (29\%) showed low expression and 23 of 51 cases (45\%) had high expression. 21 of 25 total "high-expression" cases (84\%) demonstrated poorlydifferentiated growth patterns, out of which, 7 cases (33\%) had a recognized pure solid growth pattern (See Table 2A, Table 2B and Table 3). Based on available follow up information about the patients' responses to FDA approved immune checkpoint inhibitors, 22 patients had started the treatment and continued to follow up in our institution. 14 patients received Pembrolizumab (Keytruda), and 8 patients received Nivolumab (Opdivo). The 1-year follow up showed 1 patient with complete response (clinically and radiologically), 2 patients with partial response, and 8 patients had disease progression while being on the treatment. The rest of the patients either were not offered or they declined this treatment, transferred their care to other institutions, had failed follow-up, or had the treatment interrupted or terminated because of side effects. The 3 reported patients with complete or partial responses had tumors with "high-expression" PD-L1 (See Table 4). The other 8 patients with disease progression had tumors showing either low or high PD-L1 expression.

\section{Discussion}

Lung adenocarcinoma subtypes have been defined by the 2011 International Association for the Study of Lung Cancer (IASLC) classification, and these criteria were adopted in the 2015 WHO classification [1]. In 


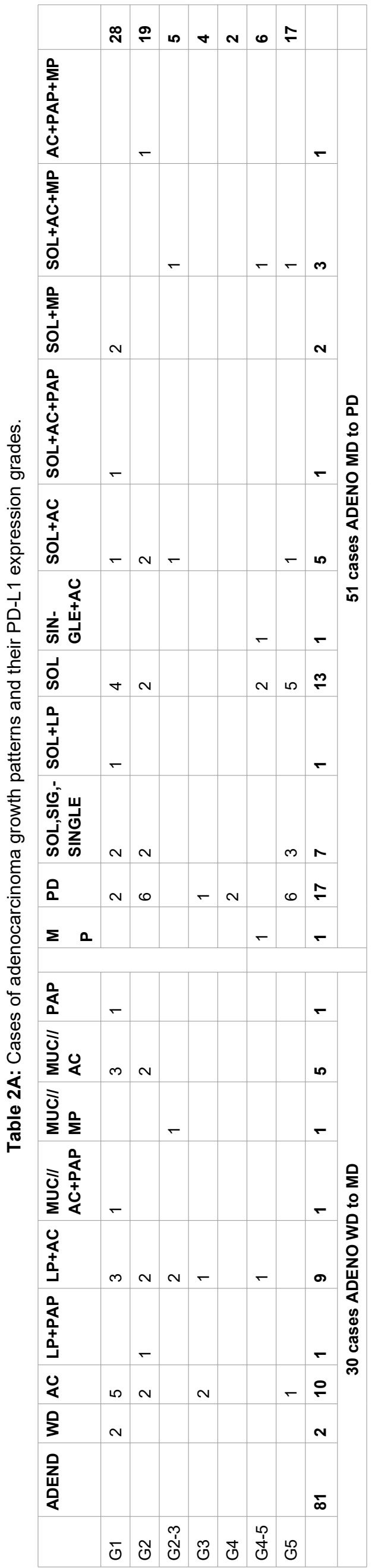

Table 2B: Cases of squamous cell carcinoma growth patterns and their PD-L1 expression grades.

\begin{tabular}{|l|l|l|l|l|}
\hline & SCC & SCC, MD, K & SCC, PD, NK & \\
\hline G1 & & 2 & 6 & 8 \\
\hline G2 & & 3 & 2 & 5 \\
\hline G2-3 & & 1 & 1 \\
\hline G3 & 1 & & 1 \\
\hline G4 & 1 & 2 & 3 \\
\hline G4-5 & & 1 & 1 \\
\hline G5 & & 1 & 1 & 2 \\
\hline & 21 & 8 & 13 & \\
\hline
\end{tabular}

SCC: Squamous Cell Carcinoma; MD: ModeratelyDifferentiated, PD: Poorly-Differentiated; K: Keratinizing; NK: Non-Keratinizing

Table 3: Combined results of adenocarcinoma cases (growth patterns and their PD-L1 expression grades) into two differentiation categories.

\begin{tabular}{|l|l|l|l|}
\hline & ADENO & WD TO MD & MD TO PD \\
\hline G1 & $28 / 81$ & $15 / 30$ & $13 / 51$ \\
\hline G2 & $19 / 81$ & $7 / 30$ & $12 / 51$ \\
\hline G2-3 & $5 / 81$ & $3 / 30$ & $2 / 51$ \\
\hline G3 & $4 / 81$ & $3 / 30$ & $1 / 51$ \\
\hline G4 & $2 / 81$ & $0 / 30$ & $2 / 51$ \\
\hline G4-5 & $6 / 81$ & $1 / 30$ & $5 / 51$ \\
\hline G5 & $17 / 81$ & $1 / 30$ & $16 / 51$ \\
\hline
\end{tabular}

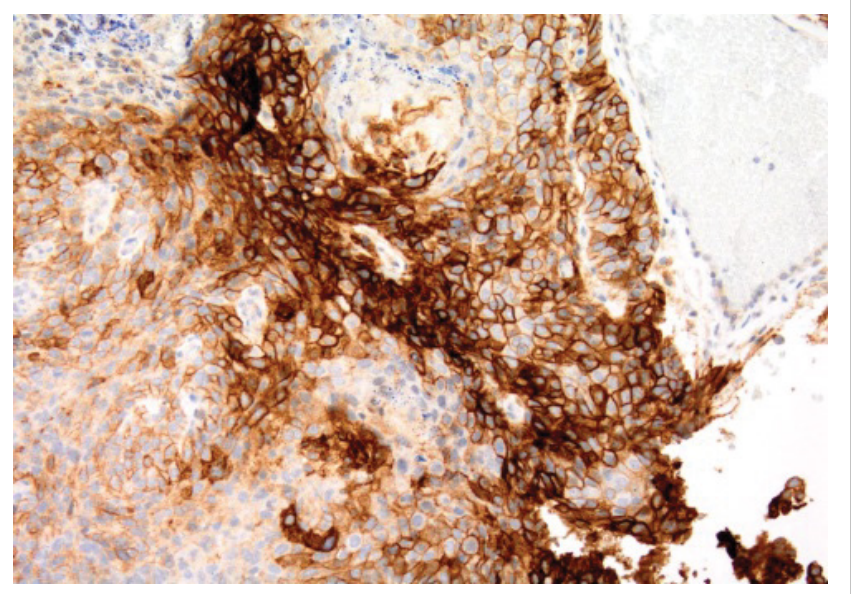

Figure 1: $66 \mathrm{~F}, \mathrm{SCC}$, keratinizing, metastatic to brain, $\mathrm{G} 4$ PD-L1.

this study, we found that majority of squamous cell carcinoma display either negative or low expression of PD-L1 protein detected by IHC, regardless of its degree of differentiation (see Figure 1). In adenocarcinomas, however, tumors with PD pattern show high PD-L1 expression, with pure solid adenocarcinoma has the highest expression level (see Figures 2 and Figure 3). The degree of expression becomes lower when combined with other growth patterns of better differentiation. On multiple cases $(n=13)$, the clinicians requested the test to be performed on small cell carcinoma, and 
Table 4: Patients' responses compared to PD-L1 expression.

\begin{tabular}{|l|l|l|l|l|}
\hline NSCLC type & Age/Gender & PD-L1 expression & Treatment given & Response \\
\hline Adenocarcinoma & $63 \mathrm{~F}$ & G2 & Nevolumab & Progression \\
\hline Adenocarcinoma & $84 \mathrm{M}$ & G5 & Nevolumab & Partial response \\
\hline Adenocarcinoma & $77 \mathrm{M}$ & G2 & Nevolumab & Progression \\
\hline Adenocarcinoma & $64 \mathrm{~F}$ & G2 & Pembrolizumab & Progression \\
\hline Adenocarcinoma & $75 \mathrm{M}$ & G4-5 & Nevolumab & Progression \\
\hline Adenocarcinoma & $86 \mathrm{~F}$ & G4-5 & Nevolumab & Partial response \\
\hline Adenocarcinoma & $49 \mathrm{~F}$ & G2 & Pembrolizumab & Progression \\
\hline Adenocarcinoma & $61 \mathrm{M}$ & G5 & Pembrolizumab & Progression \\
\hline Adenocarcinoma & $64 \mathrm{M}$ & G3 & Pembrolizumab & Progression \\
\hline SCC & $85 \mathrm{~F}$ & G4 & Pembrolizumab & Progression \\
\hline Adenocarcinoma & $61 \mathrm{M}$ & G5 & Pembrolizumab & Complete response \\
\hline
\end{tabular}

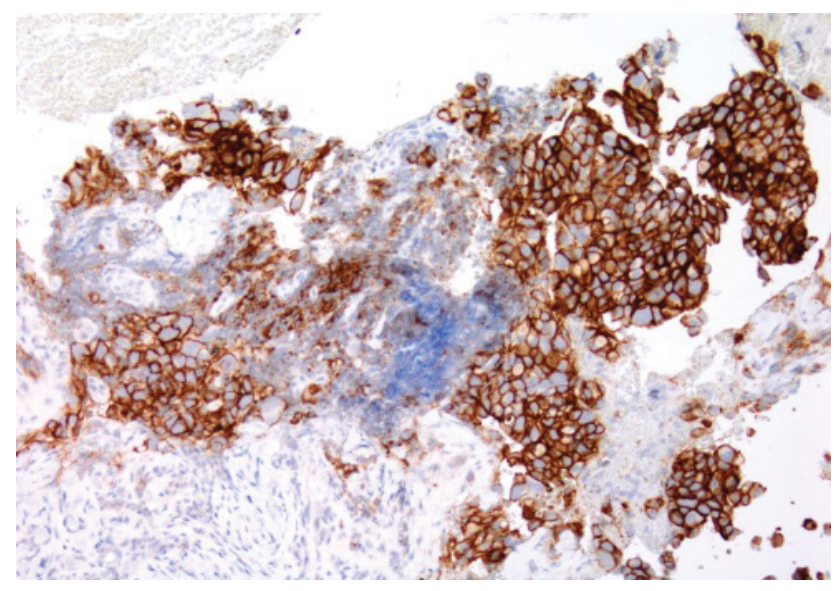

Figure 2: $62 \mathrm{~F}$, Adenocarcinoma, solid pattern, metastatic to LN,G5 PD-L1.

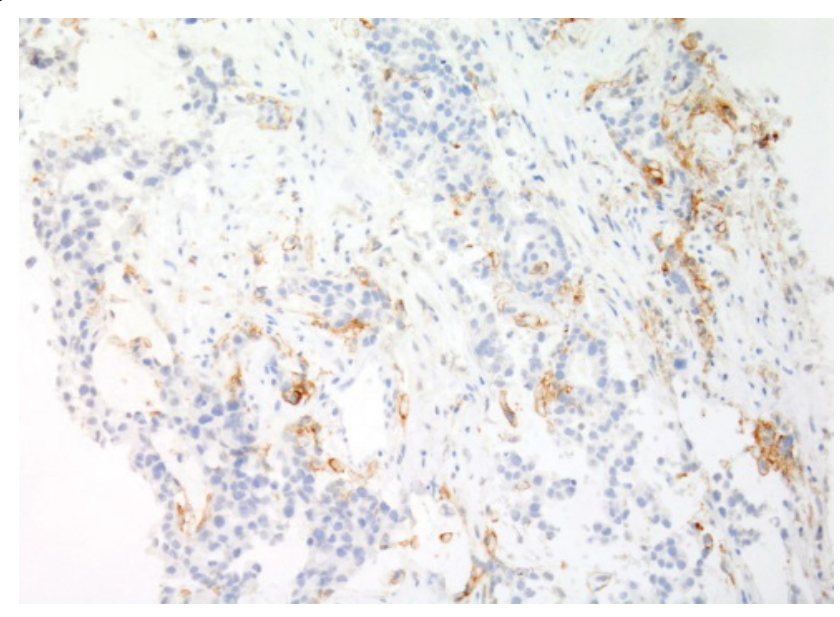

Figure 3: $58 \mathrm{~F}$, Adenocarcinoma, acinar pattern, metastatic to LN,G2 PD-L1.

they all showed negative expression of PD-L1 [2,3]. As intratumoral inflammatory cells also express PD$\mathrm{L} 1$, one should be cautious when interpreting PD-L1 expression to avoid false positive calls (see Figure 4). A known limitation to this study is we evaluated the PD-L1 expression only on the available material tested, which in most instances was either a cell block from cytology specimen, or a core needle biopsy. The available

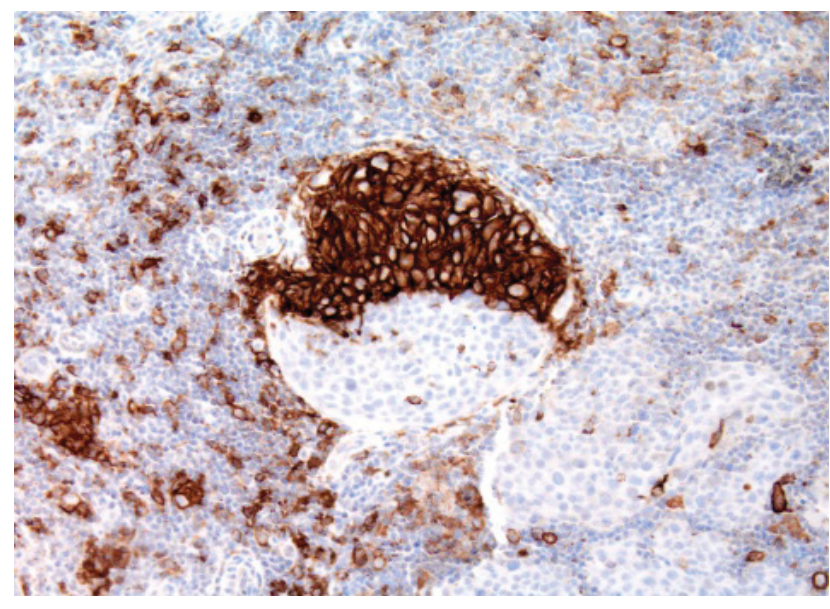

Figure 4: $54 \mathrm{~F}, \mathrm{SCC}$, keratinizing, metastatic to LN. All strongly positive staining cells are peritumoral macrophages, rather than tumor cells.

material might not accurately reflect the full picture of the entire tumor, especially if it is a tumor with mixed growth patterns, but also keeping in consideration that in such advanced disease with metastases the initial tested material will not be followed by a generous resection, and even with a wedge resection or lobectomy specimens, the test was never repeated. However, some studies have shown a strong correlation between PD-L1 expression in cytologic and surgical specimens [4]. The value of this study is to provide some opinion about the behavior of the tumor cells in regards to the degree of PD-L1 expression as early as the H\&E slides examination level, which could be of some relevance to the clinical team. The other value is to evaluate the response of the patient to the immunotherapy provided when the tumor has mixed growth patterns, predicting that some resistance or ineffectiveness might be related to other components of the tumor. Neuroendocrine neoplasms of the lung, as a component of non-small cell lung cancer, were not investigated in this study. A recent study [1] studied the expression of PD-L1 compared to clinicopathologic features of tumor cells, as well as tumor infiltrating immune cells, using SP142 clone. To our knowledge, this is the first study to be performed 
on a well-rounded variety of specimens, including cytologic and tissue biopsy specimens, with sources varying between primary lung and metastatic sites. It is also the first study run in a big cancer institution using the FDA approved 22C3 clone antibody and the FDA approved Dako 48 autostainer. The correlation of PD-L1 expression with histologic grade and solid subtype may be clinically useful. Currently, frontline use of Pembrolizumab (Keytruda) in advanced lung cancer is limited to patients with high expression of PD-L1 [1]. In patients with NSCLC showing solid growth pattern or with other patterns of poor differentiation, initiating treatment early will be of benefit. In patients with a small biopsy that is negative for PD-L1 expression but with clinicopathologic features that would suggest a high probability of PD-L1 expression, re-biopsy might be considered to assure that patients who are candidates for frontline immune checkpoint inhibitors are not missed [1]. As for the correlation with available immune checkpoint inhibitors, although we have few patients with reported complete and partial responses who had tumors with "high-expression" PD-L1, it is still early to evaluate the full advantage of treatment in these patients. However, noticing good responses in patients with high-expression tumors gives hope to more patients, and indicates that more research is still needed in this direction.

\section{References}

1. Driver BR, Miller RA, Miller $T$, Deavers $M$, Gorman B, et al. (2017) Programmed Death Ligand-1 (PD-L1) Expression in Either Tumor Cells or Tumor-Infiltrating Immune Cells Correlates With Solid and High-Grade Lung Adenocarcinomas. Arch Pathol Lab Med 141: 1529-1532.

2. Kerr KM, Nicolson MC (2016) Non-Small Cell Lung Cancer, PD-L1, and the Pathologist. Arch Pathol Lab Med 140: 249254.

3. Yu H, Boyle TA, Zhou C, Rimm DL, Hirsch FR (2016) PD-L1 Expression in Lung Cancer. J Thorac Oncol 11: 964-975.

4. Russell-Goldman E, Kravets S, Dahlberg SE, Sholl LM (2018) Cytologic-histologic correlation of programmed death-ligand 1 immunohistochemistry in lung carcinomas: PD-L1 Staining in Cytology Specimens. Cancer Cytopathol 126: $253-263$. 